\title{
The Role of Electric Field and Ultrasonication in the Deposition and Alignment of Single-Walled Carbon Nanotube Networks Using Dielectrophoresis
}

\author{
S. Ammu, D. Heskett
}

Department of Physics, University of Rhode Island, Kingston, USA.

Email: dheskett@uri.edu

Received July $14^{\text {th }}, 2013$; revised August $19^{\text {th }}, 2013$; accepted September $2^{\text {nd }}, 2013$

Copyright (C) 2013 S. Ammu, D. Heskett. This is an open access article distributed under the Creative Commons Attribution License, which permits unrestricted use, distribution, and reproduction in any medium, provided the original work is properly cited.

\begin{abstract}
The effects of electric field and ultrasonication on the deposition and alignment of single-walled carbon nanotubes (SWCNTs) across a $10 \mu \mathrm{m}$ electrode gap have been studied. It was found that a frequency of $\sim 1 \mathrm{MHz}$ of the applied field yields the largest current independent of the magnitude of the voltage or the ultrasonication time of the sample. Increasing the ultrasonication time of a SWCNT solution changes the I-V characteristics of the deposited nanotubes from linear to nonlinear for all the voltages and frequencies of the applied field. Even in the absence of an electric field, SWCNTs bridged the electrode gap up to a critical sonication time which depends on the concentration of nanotubes in the solution.
\end{abstract}

Keywords: Carbon Nanotubes; Dielectrophoresis; Ultrasonication

\section{Introduction}

Carbon nanotubes (CNTs) are cylindrically wrapped graphene sheets with unique electrical, mechanical and thermal properties [1-3]. In order to obtain devices with CNTs as the major component, it is essential to be able to align the nanotubes in a desired direction. Dielectrophoresis (DEP) is a simple yet versatile technique that has been used to obtain aligned networks and individual carbon nanotubes [4-7]. Several parameters influence the alignment of nanotubes including the voltage and frequency of the applied field, the solvents in which the CNTs are dispersed, and the ultrasonication time of the CNT solution.

Dielectrophoresis is a process in which micro/nano particles align in an applied electric field. The DEP force on a cylindrical particle with the long axis aligned parallel to the electric field can be written as [8]:

$$
F_{D E P} \propto \varepsilon_{m} \operatorname{Re}\left[\frac{\varepsilon_{p}^{*}-\varepsilon_{m}^{*}}{\varepsilon_{m}^{*}}\right] \nabla|E|^{2}
$$

where $\varepsilon_{p}^{*}$ and $\varepsilon_{m}^{*}$ are the complex permittivities of the particle and medium respectively and $E$ is the magnitude of the electric field. If the permittivity of the particle is greater (less) than the medium, the DEP force on the particle is positive (negative), which results in the movement of the particle to regions of high (low) electric field density. CNTs can be metallic or semiconducting. Since the permittivity of metallic CNTs is very high, the metallic tubes always experience a positive DEP force and are therefore attracted to the electrode edges where the field intensity is maximum. Semiconducting tubes however may experience a negative DEP at high frequencies depending on the permittivity of the medium.

A bundle of CNTs typically contains both metallic and semiconducting carbon nanotubes. In such a case, when an electric field is applied between an adjacent pair of electrodes, the nanotube bundle is attracted to the electrode edge independent of the frequency of the applied field because the permittivity of the bundle is dominated by that of the metallic tubes present in it [9].

Ultrasonicating a solution of CNTs is commonly used to deaggregate the bundled tubes by breaking the van der Waals forces that keep them together and to disperse the CNTs in the solution [10-13]. However such a process also reduces the lengths of the nanotubes present in the solution [14-16]. Smaller tubes that experience a positive DEP force tend to get attracted to the electrode edge and 
acquire the potential of the electrode. Other tubes may then connect to the edge-attached tubes. This process results in a chain which may form an electrical connection between electrodes even if the electrode gap size is greater than the length of the nanotubes.

The effects of varying the voltage of the applied field and concentration of nanotubes on the DEP alignment of single-walled carbon nanotubes (SWCNTs) has been studied previously [17-20]. It was found that lower voltages result in higher resistances across the electrode gap [17]. In this paper, we study the effects of frequency of the applied field on the DEP of SWCNTs for several applied voltages. The effect of ultrasonication times of the SWCNT solution on the alignment is also studied both in the absence and presence of an electric field.

\section{Experimental Details}

The SWCNT samples were obtained from Cheap Tubes Inc with a $90 \mathrm{wt} \%$ purity. The SWCNTs were suspended in deionized (DI) water with polyvinylpyrrolidone (PVP) as the surfactant. The nanotubes were $1-2 \mathrm{~nm}$ in diameter and $5-30 \mu \mathrm{m}$ long. The original concentration of the solution was $2 \mathrm{~g} / \mathrm{L}$. The solution was a mix of both metallic and semiconducting nanotubes containing approximately $1 / 3$ metallic and $2 / 3$ semiconducting tubes [4,9]. The original solution was diluted with DI water to various concentrations. A Branson 1510 bath sonicator was used to carry out the ultrasonication of the SWCNT sample. The samples were maintained at room temperature throughout the study.

A pair of indium tin oxide (ITO) electrodes deposited on a glass substrate was used for the electrical measurements. The electrodes were obtained from Abtech Scientific Inc. The thickness of the metal was $1000 \AA$. The electrode spacing was $10 \mu \mathrm{m}$, the width of the electrode was $3 \mu \mathrm{m}$ and the length of each electrode was $3 \mathrm{~mm}$.

During the SWCNT deposition, an AC electric field was applied with a Wavetek $20 \mathrm{MHz}$ pulse function generator connected to a Tektronix oscilloscope. Approximately $5 \mu \mathrm{l}$ of the SWCNT solution was dropped on the electrodes with either the field on or off. If the electric field was turned on, the voltage across the gap was monitored in real time until the sample dried at room temperature. If the SWCNTs align along the direction of the field and bridge the gap between the electrodes, a voltage drop across the gap is observed.

After the solution dried on the electrodes, the electrodes were connected to a DC power supply with an ammeter in series. The voltage across the electrodes was varied from $-1.5 \mathrm{~V}$ to $1.5 \mathrm{~V}$ while the current was measured. The I-V curves were plotted in Logger Pro and the conductance was obtained by linear fits to the graphs.

It should be noted that the terms "field frequency" and "field" in the remainder of this article refer to the frequency and the AC field applied between the electrodes during the DEP process to align the SWCNTs, but not during the electrical measurements.

\section{Results and Discussion}

\subsection{Results without an Electric Field}

In order to determine if the nanotubes will bridge the electrode gap in the absence of a field, SWCNT samples were deposited on the electrodes without any application of the field and allowed to dry at room temperature. The original sample was diluted to various concentrations $(1 / 2-1 / 64$ of the original concentration of $2 \mathrm{~g} / \mathrm{L})$ and I-V data were taken for each concentration. Figure 1 shows a plot of the current as a function of the ultrasonication time at a voltage of $1.3 \mathrm{~V}$ for a $1 / 15$ concentration. For $1 / 15$ and lower concentrations, the current decreased monotonically with the increase in ultrasonication time. In contrast, for concentrations greater than $1 / 15$ of original, it was found that the current followed a non-monotonous variation as a function of the ultrasonication time.

Figure 2 shows the I-V curves plotted for a 1/64 concentration for different ultrasonication times of the SWCNT solution. The I-V curves are nonlinear for all ultrasonication times. In addition, we did not detect any current across the gap beyond a critical ultrasonication time of 210 minutes for this particular concentration. This critical ultrasonication time increased with the concentration of the nanotubes in the solution. This is shown in Figure 3 where the critical ultrasonication time is plotted as a function of the nanotube concentration.

The lengths of the SWCNTs obtained originally ranged from $5 \mu \mathrm{m}$ to $30 \mu \mathrm{m}$ while the electrode gap was $10 \mu \mathrm{m}$.

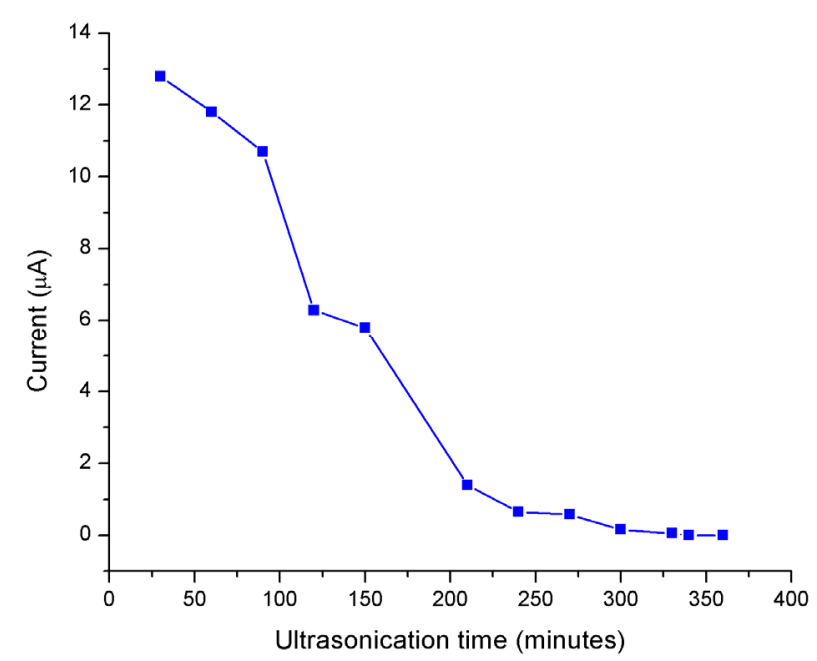

Figure 1. The currrent as a function of ultrasonication time at $1.3 \mathrm{~V}$ for a $1 / 15$ th concentration. 


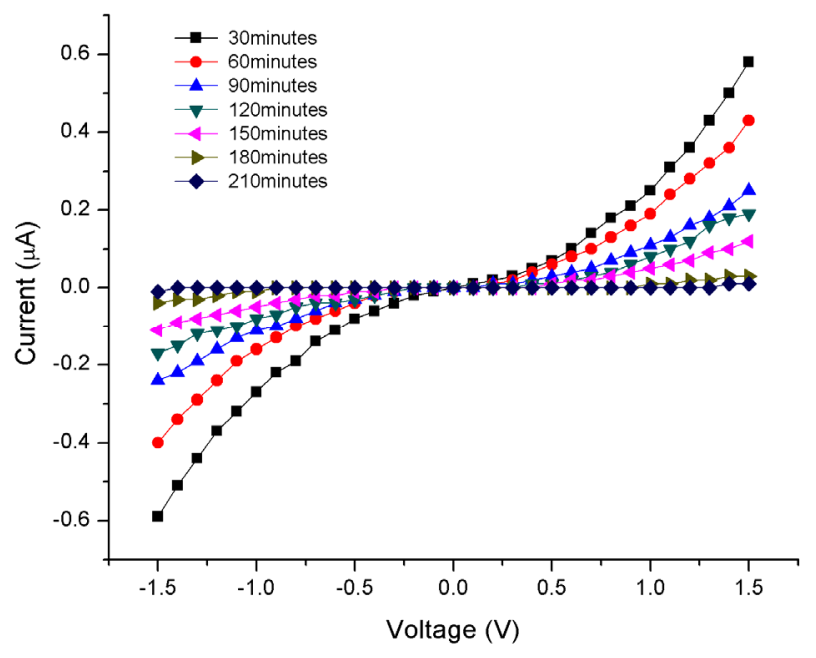

Figure 2. Plot of the currrent vs voltage of a 1/64th concentration sample for different ultrasonication times in the absence of a field.

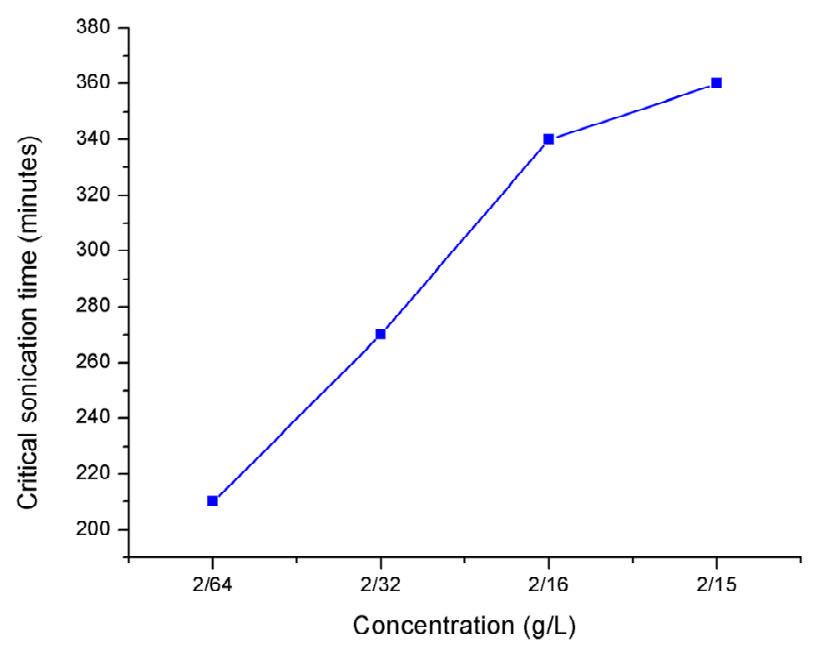

Figure 3. The critical ultrasonication time (the ultrasonication time beyond which no current was detected across the gap) as a function of the concentration.

When no electric field was applied, the nanotubes deposit randomly between the electrodes but bridge the gap between them since the lengths of many of the tubes are greater than the width of the interelectrode gap. However as the sample is sonicated longer, the nanotubes are shortened in length and are unable to bridge the gap, resulting in no current. The critical ultrasonication time is important since it is desirable in some applications that the nanotubes align and bridge the gaps due to the DEP process but not due to longer lengths of the nanotubes.

Since there are a larger fraction of semiconducting tubes in solution ( $\sim 2 / 3$ semiconducting vs. $1 / 3$ metallic), we suggest that the non-linearity in the I-V curves (Figure 2) for any ultrasonication time is due to the larger fraction of semiconducting tubes that deposit randomly between the electrodes.

\subsection{Results with an Electric Field}

\subsubsection{Ultrasonication Time Dependence}

In order to test the dependence of ultrasonication time in the presence of a field, the electrical properties of a 1/64 concentration sample were examined as the ultrasonication time was varied from 15 minutes to 75 minutes. Figure 4 shows the I-V characteristics of a dielectrophoretically aligned sample using a $10 \mathrm{~V}$ peak to peak voltage. The current decreased monotonically with the increase in ultrasonication time. In addition, the I-V curves were linear up to an ultrasonication time of 30 minutes, indicating ohmic contacts at the electrodes and across the gap. As the ultrasonication time increased beyond 30 minutes, the I-V curves start to show nonlinear characteristics with the nonlinearity increasing with the ultrasonication time. The change to nonlinearity occurred at all the frequencies and voltages of the applied field. This change from linearity to nonlinearity occurred at $45 \mathrm{~min}$ utes for a field of $15 \mathrm{~V}$ and 60 minutes for $19 \mathrm{~V}$. This is in contrast to the results obtained without an electric field in which case the I-V curves exhibit nonlinearity for all ultrasonication times (Figure 2), as discussed above.

We propose that the change from linearity to nonlinearity in the presence of an electric field is due to two reasons: A bundle of CNTs most likely contains at least one metallic tube and hence the permittivity of the bundle is dominated by the metallic tube [9]. At lower ultrasonication times, this bundle experiences a positive DEP force independent of the frequency of the applied field, leading to the bridging of the gap. As the ultrasonication time increases, the nanotube bundle is not only separated

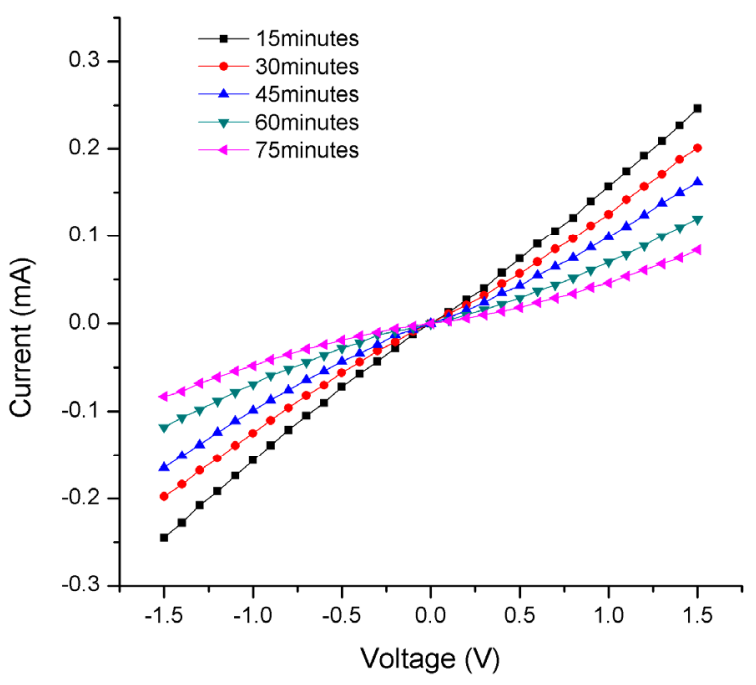

Figure 4. Plot of current vs voltage for a 1/64th concentration at different ultrasonication times. A $10 \mathrm{~V}$ peak-peak AC voltage was applied at a frequency of $15 \mathrm{MHz}$. 
but it also gets shortened and this leads to fewer tubes bridging the gap thereby leading to a decrease in current. The interelectrode gap is now bridged by a set of semiconducting tubes or a mix of metallic and semiconducting tubes. This results in the nonlinear characteristic of the I-V curves (similar to the nonlinearity observed in all cases when no electric field was present). In an investigation in which multiwalled carbon nanotubes (MWCNTs) were aligned with an electric field, Cicoria and Sun [21] observed a nonlinearity in the I-V curves that is similar to our results. They suggested that the presence of a single short semiconducting CNT in the network of nanotubes results in a nonlinear behaviour which agrees with our interpretation.

At higher supply voltages, the SWCNTs experience more DEP force on them, favoring the attraction of the metallic CNTs to the electrodes. Hence longer ultrasonication times (resulting in shorter tubes on average) are required for the change to nonlinearity as the voltage is increased.

\subsubsection{Frequency Dependence}

We also tested the dependence of the frequency of the applied field on the DEP process. With a 1/64th concentration, the frequency of the applied field was varied from $100 \mathrm{KHz}$ to $20 \mathrm{MHz}$. As an example, in Figure 5 is plotted the conductance $(\mathrm{mA} / \mathrm{V})$ obtained from the linear fits as a function of the field frequency for several voltages. For all these examples and for all the concentrations used in our study, the conductance was found to be the highest at $\sim 1 \mathrm{MHz}$.

We suggest that the SWCNTs cannot keep up with the rapid oscillations at high frequencies of the AC field. Hence the SWCNTs do not have enough time to reach

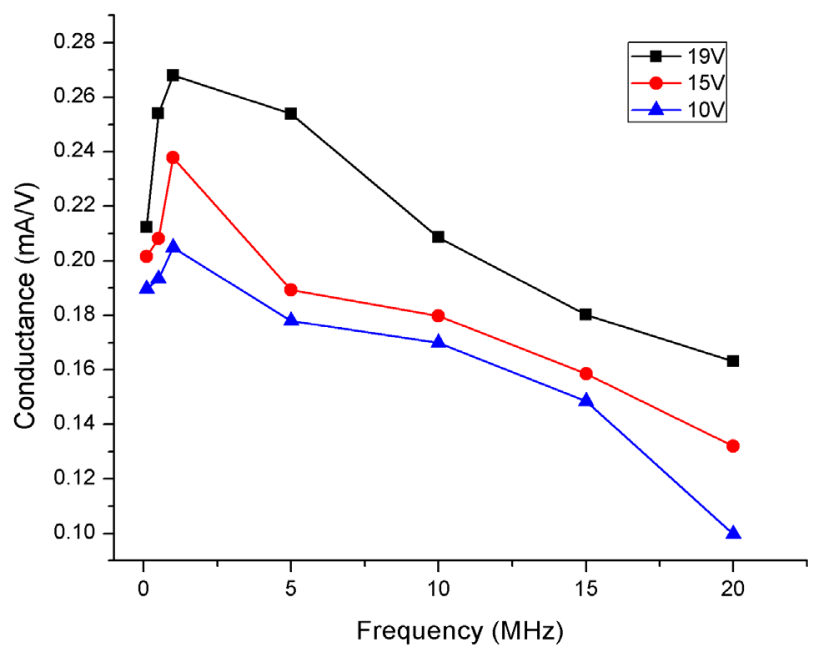

Figure 5. The conductance as a function of field frequency for three voltages $(19 \mathrm{~V}, 15 \mathrm{~V}$, and $10 \mathrm{~V})$. A 1/64th concentration sample was sonicated for 30 minutes. the electrodes and align along the gap before the solution evaporates, resulting in lower conductance at higher frequencies. A similar result was reported by Moscatello et al. on MWCNTs [22].

\section{Summary and Conclusions}

The effects of ultrasonication and electric field on the DEP alignment of SWCNTs has been studied. In the presence of an electric field, the characteristics of the SWCNTs aligned in the DEP process depends strongly on the ultrasonication time of the SWCNT solution. Increasing the ultrasonication time changes the device characteristics from linear to nonlinear for all voltages and frequencies of the field. The conductance was found to be frequency dependent with the conductance being maximum at lower frequencies, typically $1 \mathrm{MHz}$. It was also found that the SWCNTs bridge the gap even in the absence of a field up to a critical ultrasonication time that depends on the concentration of SWCNTs in the solution.

\section{REFERENCES}

[1] S. Kim, S. Y. Xuan, P. D. Ye, S. Mohammadi and S. W. Lee, "Single-Walled Carbon Nanotube Transistors Fabricated by Advanced Alignment Techniques Utilizing CVD Growth and Dielectrophoresis," Solid-State Electronics, Vol. 52, No. 8, 2008, pp. 1260-1263. http://dx.doi.org/10.1016/j.sse.2008.05.003

[2] M. Meyyappan, "Carbon Nanotubes: Science and Applications," CRC Press, 2005.

[3] P. J. F. Harris, “Carbon Nanotube Science: Synthesis, Properties and Applications," Cambridge University Press, Cambridge, 2009. http://dx.doi.org/10.1017/CBO9780511609701

[4] M. Dimaki and P. Boggild, "Waferscale Assembly of Field-Aligned Nanotube Networks (FANs)," Physica Status Solidi A, Vol. 203, No. 6, 2006, pp. 1088-1093. http://dx.doi.org/10.1002/pssa.200566172

[5] M. Riegelman, H. Liu and H. H. Bau, "Controlled Nanoassembly and Construction of Nanofluidic Devices," Journal of Fluids Engineering, Vol. 128, No. 1, 2006, pp. 613. http://dx.doi.org/10.1115/1.2136932

[6] S. Taeger, D. Sickert, P. Atanasov, G. Eckstein and M. Mertig, "Self-Assembly of Carbon Nanotube Field-Effect Transistors by Ac-Dielectrophoresis," Physica Status Solidi B, Vol. 243, No. 13, 2006, pp. 3355-3358. http://dx.doi.org/10.1002/pssb.200669182

[7] L. M. Huang, Z. Jia and S. O'Brien, "Orientated Assembly of Single-Walled Carbon Nanotubes and Applications," Journal of Materials Chemistry, Vol. 17, No. 37, 2007, pp. 3863-3874. http://dx.doi.org/10.1039/b702080e

[8] M. Dimaki and P. Bøggild, "Dielectrophoresis of Carbon Nanotubes Using Microelectrodes: A Numerical Study," Nanotechnology, Vol. 15, No. 8, 2004, p. 1095. 
http://dx.doi.org/10.1088/0957-4484/15/8/039

[9] R. Krupke, F. Hennrich, H. V. Löhneysen and M. M. Kappes, "Separation of Metallic from Semiconducting Single-Walled Carbon Nanotubes," Science, Vol. 301, No. 5631, 2003, pp. 344-347. http://dx.doi.org/10.1126/science.1086534

[10] M. J. O'Connell, S. M. Bachilo, C. B. Huffman, V. C. Moore, M. S. Strano, E. H. Haroz, et al., "Band Gap Fluorescence from Individual Single-Walled Carbon Nanotubes," Science, Vol. 297, No. 5581, 2002, pp. 593-596. http://dx.doi.org/10.1126/science.1072631

[11] S. Niyogi, M. A. Hamon, D. E. Perea, C. B. Kang, B. Zhao, S. K. Pal, et al., "Ultrasonic Dispersions of SingleWalled Carbon Nanotubes," Journal of Physical Chemistry B, Vol. 107, No. 34, 2003, pp. 8799-8804. http://dx.doi.org/10.1021/jp034866d

[12] A. Koshio, M. Yudasaka, M. Zhang and S. Iijima, "A Simple Way to Chemically React Single-Wall Carbon Nanotubes with Organic Materials Using Ultrasonication," Nano Letters, Vol. 1, No. 7, 2001, pp. 361-363. http://dx.doi.org/10.1021/n10155431

[13] J. Hilding, E. A. Grulke, Z. G. Zhang and F. Lockwood, "Dispersion of Carbon Nanotubes in Liquids," Journal of Dispersion Science and Technology, Vol. 24, No. 1, 2003, pp. 1-41. http://dx.doi.org/10.1021/n10155431

[14] K. B. Shelimov, R. O. Esenaliev, A. G. Rinzler, C. B. Huffman and R. E. Smalley, "Purification of Single-Wall Carbon Nanotubes by Ultrasonically Assisted Filtration," Chemical Physics Letters, Vol. 282, No. 5-6, 1998, pp. 429-434. http://dx.doi.org/10.1016/S0009-2614(97)01265-7

[15] D. A. Heller, R. M. Mayrhofer, S. Baik, Y. V. Grinkova, M. L. Usrey and M. S. Strano, "Concomitant Length and Diameter Separation of Single-Walled Carbon Nanotubes," Journal of the American Chemical Society, Vol.
126, No. 44, 2004, pp. 14567-14573.

http://dx.doi.org/10.1021/ja046450z

[16] K. Fu and Y. P. Sun, "Dispersion and Solubilization of Carbon Nanotubes," Journal of Nanoscience and Nanotechnology, Vol. 3, No. 5, 2003, pp. 351-364. http://dx.doi.org/10.1166/jnn.2003.225

[17] M. Ouyang, M. L. Y. Sin, G. C. T. Chow, W. J. Li, X. Han and D. C. Janzen, "DEP-Based Fabrication and Characterization of Electronic-Grade CNTs for Nano-Sensing Applications," Proceedings of the 7th IEEE Conference on Nanotechnology, 2007, pp. 1-6.

[18] S. Banerjee, B. E. White, L. Huang, B. J. Rego, S. O'Brien and I. P. Herman, "Precise Positioning of Single-Walled Carbon Nanotubes by Ac Dielectrophoresis," Journal of Vacuum Science \& Technology B, Vol. 24, No. 6, 2006, p. 3173. http://dx.doi.org/10.1116/1.2387155

[19] A. Subramanian, B. Vikramaditya, L. Dong, D. Bell and B. J. Nelson, "Micro and Nanorobotic Assembly Using Dielectrophoresis," Proceedings of Robotics: Science and Systems, Cambridge, 2005, pp. 208-215.

[20] P. Li and W. Xue, "Selective Deposition and Alignment of Single-Walled Carbon Nanotubes Assisted by Dielectrophoresis: From Thin Films to Individual Nanotubes," NanoScale Research Letters, Vol. 5, 2010, pp. 1072-1078. http://dx.doi.org/10.1007/s11671-010-9604-3

[21] R. Cicoria and Y. Sun, "Dielectrophoretically Trapping Semiconductive Carbon Nanotube Networks," Nanotechnology, Vol. 19, No. 48, 2008, Article ID: 485303. http://dx.doi.org/10.1088/0957-4484/19/48/485303

[22] J. Moscatello, V. Kayastha, B. Ulmen, A. Pandey, S. Wu, A. Singh, et al., "Surfactant-Free Dielectrophoretic Deposition of Multi-Walled Carbon Nanotubes with Tunable Deposition Density," Carbon, Vol. 48, No. 12, 2010, pp. 3559-3569.

http://dx.doi.org/10.1016/j.carbon.2010.05.054 